\title{
Linking fluvial bed sediment transport across scales
}

\author{
Yong Zhang, ${ }^{1}$ Mark M. Meerschaert, ${ }^{2}$ and Aaron I. Packman ${ }^{3}$ \\ Received 6 August 2012; revised 11 September 2012; accepted 12 September 2012; published 23 October 2012.
}

[1] We present a new random walk model for bed load sediment transport that explains the scale-dependency generally observed in transport rates and captures the transient anomalous dispersion often seen in rivers. Particles alternate between mobile and resting phases, with a tempered stable probability distribution for both particle step length and resting time. Tempered fractional mobile-immobile differential equations model the ensemble average of particle dynamics. The model is tested against data from three sediment dispersion experiments. Using tempering in both space and time, the new model is able to capture the full range of observed ensemble particle dynamics. The random walk model illuminates the physical meaning of all transport parameters in the mobile-immobile equations and explains transitions between observed super-diffusive, sub-diffusive, and regular diffusive ensemble particle dynamics. By explicitly predicting the effects of spatial and temporal averaging on particle dynamics, this method can be used to link observations of fluvial sediment dynamics across scales. This approach is also generally applicable to a wide variety of geophysical and ecological dynamics, such as ecological dispersal, pathogen transmission in rivers, nutrient export from watersheds, and large-scale geomorphodynamics associated with infrequent phenomena such as avalanches and turbidity currents. Citation: Zhang, Y., M. M. Meerschaert, and A. I. Packman (2012), Linking fluvial bed sediment transport across scales, Geophys. Res. Lett., 39, L20404, doi:10.1029/2012GL053476.

\section{Introduction}

[2] Bed sediment transport in rivers is scale-dependent, with anomalous scaling observed at early-to-intermediate time scales [Jerolmack, 2011; Nikora et al., 2002]. In regular dispersion (Fickian), plumes are symmetric and plume variance grows proportionally to elapsed time. Einstein [1937] developed a random walk model for fluvial sediment transport assuming that particle transport events are independent, and both particle jump length and resting time distributions have finite moments. These conditions lead to regular dispersion. However, particle inertia contributes to initial correlated ballistic motion, and hydrodynamic forces can accelerate mobile particles, resulting in super-dispersion where the

\footnotetext{
${ }^{1}$ Division of Hydrologic Sciences, Desert Research Institute, Las Vegas, Nevada, USA.

${ }^{2}$ Department of Statistics and Probability, Michigan State University, East Lansing, Michigan, USA.

${ }^{3}$ Department of Civil and Environmental Engineering, Northwestern University, Evanston, Illinois, USA.

Corresponding author: Y. Zhang, Division of Hydrologic Sciences, Desert Research Institute, 755 East Flamingo Rd., Las Vegas, NV 89119, USA. (Yong.Zhang@dri.edu)

C2012. American Geophysical Union. All Rights Reserved. 0094-8276/12/2012GL053476
}

observed variance grows faster than linearly [Martin et al., 2012]. Bradley et al. [2010] extended Einstein's model to include super-dispersion, and showed that mobile particles remain super-diffusive over long timescales in sand-bed rivers. Particles can also be trapped in the bed, leading to subdispersion [Nikora et al., 2002]. Trapping timescales range from minutes for turnover of small bedforms to thousands of years for meander migration [Aalto et al., 2008]. On sufficiently long time scales, averaging over the range of variability in particle motion causes a relaxation to regular dispersive behavior. Based on these considerations, we propose three distinct sediment transport regimes: the early time scale, where particles exhibit super-diffusion; the intermediate scale, during which particles exhibit sub-diffusion; and the late time scale with Fickian dispersion.

[3] Here we provide a unified theory for bed sediment transport dynamics in the form of a tempered space-time random walk model. Tempered power law jumps and waiting times lead to a tempered fractional mobile-immobile model that captures particle dynamics in all three transport regimes as well as transitions between regimes (section 2). The microscopic random walk model is used to assign a specific physical meaning to each parameter in the macroscopic equations describing ensemble particle motion. We use this model to unify prior observations of laboratory- and fieldscale bed sediment transport dynamics, and show that previously observed scale dependency in particle dispersion behavior is a natural consequence of averaging of the underlying particle motion (section 3 ). We then discuss the main factors responsible for anomalous transport, the implications for interpreting observations of sediment transport at different scales, and the general utility of the approach for related geophysical and ecological problems (section 4).

\section{Methodology}

[4] The proposed microscopic model for sediment transport traces the path of each individual particle in space and time:

$$
\begin{gathered}
x_{n}=x_{n-1}+V d t+\delta x_{n}, \\
t_{n}=t_{n-1}+d t+\delta t_{n} .
\end{gathered}
$$

Here $x_{n}$ is the position of a randomly selected particle after $n$ steps, and $t_{n}$ is the time at which the particle reaches location $x_{n}$. The parameter $V$ denotes particle velocity, $d t$ is the basic time step, the random variable $\delta x_{n}$ is the $n$th dispersive displacement, and the random variable $\delta t_{n}$ is the resting time prior to the $n$-th jump. Equations (1a) and (1b) describe a Markov process in space-time. The space process (1a) accounts for sediment displacement, which can include saltation, rolling, or sliding. The time process (1b) records motion time $d t$ and time between transport events 
$\delta t_{n}$, presuming that transport is episodic as a result of both flow variations and particle trapping or burial.

[5] Einstein [1937] and Nikora et al. [2002] assumed an exponential distribution of particle step lengths, $\delta x$, while Bradley et al. [2010] replaced this with a power law $P(\delta x) \propto(\delta x)^{-\alpha}$ with $1<\alpha<2$. Conversely, Einstein [1937] and Bradley et al. [2010] assumed an exponential distribution of particle resting times, $\delta t$, while Nikora et al. [2002] used a power law $P(\delta t) \propto(\delta t)^{-v}$ with $0<v<2$. All cases lead to a tempered stable distribution in the limit [Meerschaert et al., 2008], which we employ here as a more general model of particle motion.

[6] Gaussian distributions with finite moments are the limits in the Central Limit Theorem (CLT). The stable distributions [Samorodnitsky and Taqqu, 1994] generalize the Gaussian distributions as the possible limits in the extended CLT, which allows infinite moments [Meerschaert and Sikorskii, 2012]. A random walk with power law jumps $P(\delta x) \propto(\delta x)^{-\alpha}$ where $0<\alpha<2$ converges to a stable with the same index $\alpha$ [Meerschaert and Sikorskii, 2012]. For exponential jumps, the limit is normal, corresponding to a stable with $\alpha=2$. When power law jumps are modified by an exponential, so that $P(\delta x) \propto(\delta x)^{-\alpha} e^{-\lambda \delta x}$ for some $\lambda>0$, the limit is tempered stable [Chakrabarty and Meerschaert, 2011]. A tempered stable process becomes $\alpha$-stable as $t \rightarrow 0$, and normal as $t \rightarrow \infty$ [Rosiński, 2007]. Since the stable spreads like $t^{1 / \alpha}$ and the normal spreads at the Fickian rate $t^{1 / 2}$, the tempered stable process $X_{t}$ models transient anomalous dispersion, i.e., dispersion that is initially anomalous and relaxes to regular dispersion at late times [Baeumer and Meerschaert, 2010]. The space-time random walk model ((1a) and (1b)) assumes

$$
\begin{gathered}
\delta x \sim \operatorname{TS}_{\alpha}\left(1,\left[-D d t \cos \left(\frac{\pi \alpha}{2}\right)\right]^{1 / \alpha}, 0, \lambda_{x}\right), \\
\delta t \sim \operatorname{TS}_{\gamma}\left(1,\left[\beta d t \cos \left(\frac{\pi \gamma}{2}\right)\right]^{1 / \gamma}, 0, \lambda_{t}\right),
\end{gathered}
$$

with dispersion coefficient $D$, dispersive scaling index $\alpha \in(1,2]$, spatial tempering parameter $\lambda_{x}$, capacity coefficient $\beta$, time scaling index $\gamma \in(0,1)$, and tempering parameter $\lambda_{t}$ in time. Since $\alpha<2$, the space process undergoes transient anomalous super-dispersion. Since the process $N_{t}=\max \left\{n: t_{n} \leq t\right\}$ that tracks the number of jumps by time $t$ grows at the rate $t^{\gamma}$ with $\gamma<1$, the tempered stable time process causes transient anomalous sub-dispersion [Meerschaert et al., 2008]. Typically, the super-dispersive behavior presents at the early time scale, and the sub-dispersive behavior manifests at an intermediate time scale. At the late time scale, both the space and time processes become essentially Gaussian, and the combined effect is a normal inverse Gaussian.

[7] The ensemble particle distribution described by the model (1a) and (1b) follows the TEmpered Space-Time Fractional Advection-Dispersion Equations (TESTFADE):

$$
\begin{gathered}
\frac{\partial P_{M}}{\partial t}+\beta \frac{\partial^{\gamma, \lambda_{t}} P_{M}}{\partial t^{\gamma, \lambda_{t}}}=-V \frac{\partial P_{M}}{\partial x}+D \frac{\partial^{\alpha, \lambda_{x}} P_{M}}{\partial x^{\alpha, \lambda_{x}}} \\
\frac{\partial P_{T}}{\partial t}+\beta \frac{\partial^{\gamma, \lambda_{t}} P_{T}}{\partial t^{\gamma, \lambda_{t}}}=-V \frac{\partial P_{T}}{\partial x}+D \frac{\partial^{\alpha, \lambda_{x}} P_{T}}{\partial x^{\alpha, \lambda_{x}}}+m_{0} \beta g(t) \delta(x)
\end{gathered}
$$

where $P_{M}(x, t)$ is the concentration of mobile particles, $P_{T}(x, t)$ represents the combined concentration of mobile and immobile particles, $\delta(\cdot)$ is the Dirac delta function, the memory function $g(t)=\int_{t}^{\infty} e^{-\lambda_{t} r} \gamma\left(\lambda_{t}\right)^{-\gamma-1} / \Gamma(1-\gamma) d r$ represents the rate at which particles exit the immobile phase ( $\Gamma$ is the Gamma function), and $m_{0}$ is the initial mass [Zhang and Meerschaert, 2011]. The tempered fractional derivative $\partial^{p, \lambda} f(x) / \partial x^{p, \lambda}$ is the function with Fourier transform $\left[(\lambda+i k)^{p}-\lambda^{p}\right] \hat{f}(k)$ for $0<p<1$, or $\left[(\lambda+i k)^{p}-\right.$ $\left.\lambda^{p}-i k p \lambda^{p-1}\right] \hat{f}(k)$ for $1<p<2$. Densities $g(x, t)$ of the tempered stable process $X_{t} \sim \operatorname{TS}_{\alpha}(1, t, 0, \lambda)$ solve the tempered fractional dispersion equation $\partial g / \partial t=\partial^{p, \lambda} g / \partial x^{p, \lambda}$ in either case [Baeumer and Meerschaert, 2010]. To account for nonstationarity, which is expected because of typical downstream variations in streamflow and channel morphology, the model parameters in (3a) and (3b) can vary with time. The model equations (3a) and ( $3 b)$ can be solved via particle tracking, by simulating a large number of independent particles governed by equations (1a) and (1b), and drawing their histogram, as in Zhang and Meerschaert [2011].

\section{Applications}

[8] Here the TESTFADE model ((3a) and (3b)) is applied to fluvial transport experiments.

\subsection{Case 1: Sand Transport in the North Loup River}

[9] Sayre and Hubbell [1965] released 40 pounds of radioactive sand with a median grain size of $0.305 \mathrm{~mm}$ across the river bed, and then monitored for 13 days over 1800 feet of channel. Shear velocity was approximately $u^{*}=6.9 \mathrm{~cm} / \mathrm{s}$ [Bradley et al., 2010]. They found that the tracer sand moved in discrete steps, alternating between motion and resting. During the resting phase, the particles were buried under migrating dunes, and episodically released by scour. A variety of factors, including turbulent flow-boundary interactions and variability in particle characteristics, contributed to randomness in particle motion. Associated bedforms were observed to have fractal patterns, as has been documented by others [Nikora et al., 1997; Jerolmack and Mohrig, 2005]. Sayre and Hubbell [1965] derived a concentration profile identical to Einstein [1937], assuming that the step distance and resting period distributions are exponential:

$$
f(\delta x)=k_{1} e^{-k_{1} \delta x} \quad \text { and } \quad f(\delta t)=k_{2} e^{-k_{2} \delta t}
$$

where $1 / k_{1}$ and $1 / k_{2}$ are the mean travel distance and mean resting time, respectively. Since the sum of independent exponential random variables follows a gamma distribution, this model predicts exponential tails in the concentration snapshots. However, the observed plumes have somewhat heavier tails since the leading edge is a straight line in a double-log plot (Figure 1). Bradley et al. [2010] modeled particle step length using an $\alpha$-stable distribution, to capture the heavy leading edge of the snapshots in Figure 1.

[10] Here, the TESTFADE model parameters in (3a) and (3b) were fitted as follows. Firstly, temporal parameters were estimated by fitting the detected tracer mass, yielding $\lambda_{t}=0.01$ hour $^{-1}$ since mobile mass stabilizes at $1 / \lambda_{t} \approx$ 100 hour as shown in Figure $2 \mathrm{~g}$, the height of the curve 

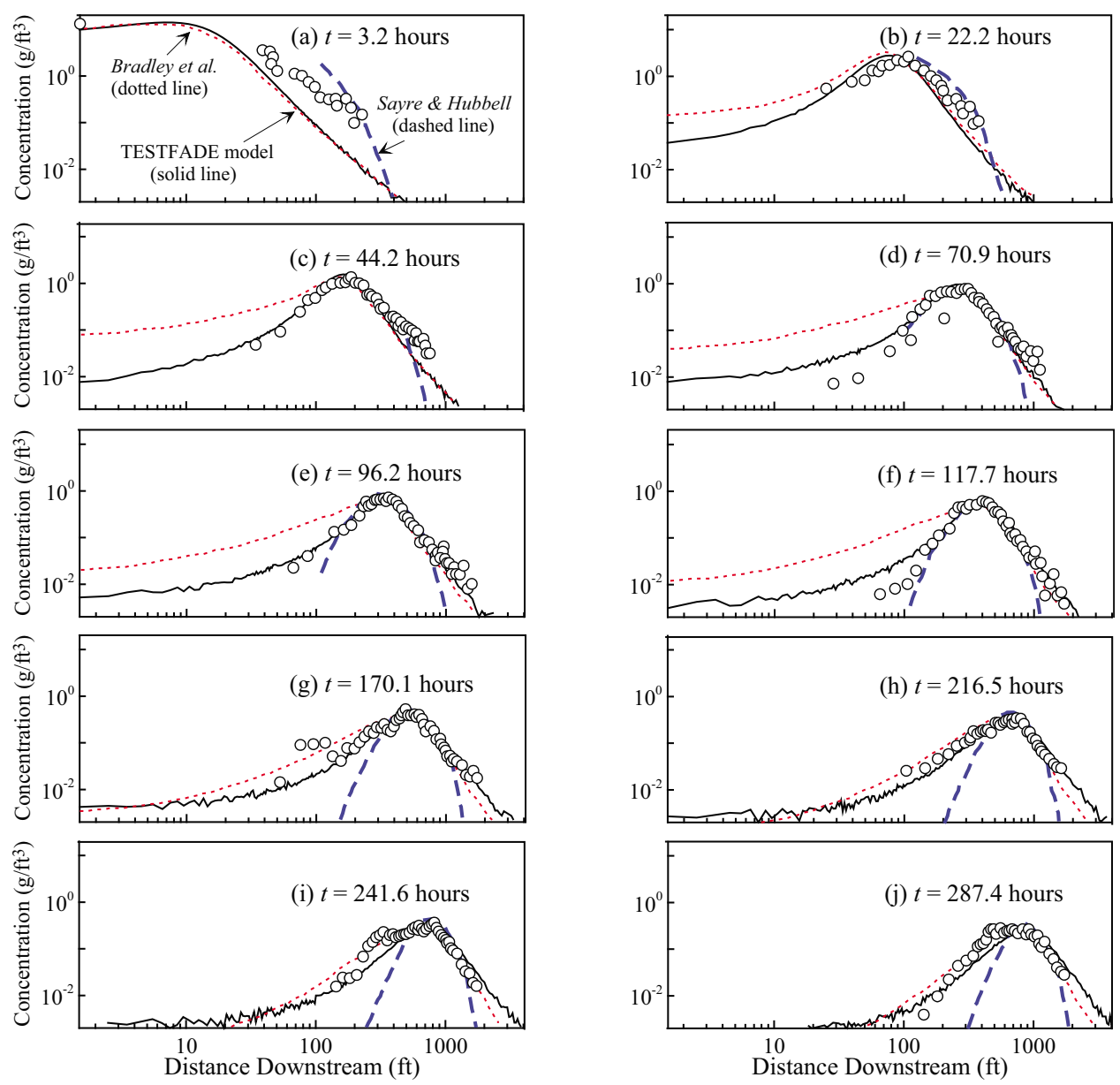

Figure 1. Concentration profiles for the mobile phase modeled by (3a) (solid line) versus measurements of Sayre and Hubbell [1965] (symbols). The model results of Bradley et al. [2010] (dotted line) and Sayre and Hubbell [1965] (dashed line) are also shown for comparison.

yielding the mobile mass ratio $\beta=0.05$ hour $^{\gamma-1}$, and the slope $M(t) \propto t^{\gamma-1}$ at early time yielding $\gamma=0.85$. Spatial parameters in (3a) and (3b) were then fit using the measured snapshots (Figure 1). First $V$ was fit to peak concentration, then $D$ to plume width, then $\alpha$ for leading edge thickness. This yields $\alpha(t)=1.30,1.10$ and 1.05 for $t<$ $60,60 \leq t<120$ and $t \geq 120 \mathrm{hr} ; D(t)=7.8,14.0$ and $20.0 \mathrm{ft}^{\alpha} / \mathrm{h}$, for the same time intervals; and $V=7.75 \mathrm{ft} / \mathrm{h}$. The tempering parameter $\lambda_{x}=0$, since the leading tail follows a power law.

[11] Compared to Bradley et al. [2010], the plume trailing tail is fitted more closely in the intermediate time range (Figure 1), because the power law retention is explicitly modeled. For these conditions, the TESTFADE model predicts super-dispersive spreading at the early time scale, with a transition to Fickian scaling at the late time scale (Figure 2). The super-dispersion at early time is consistent with the heavy tails in Figure 1, since power-law steps lead to anomalous super-dispersion.

\subsection{Case 2: Bed Load Transport in Duck Creek}

[12] Drake et al. [1988] filmed the trajectories of 125 painted 4-8 $\mathrm{mm}$ particles on the bed of Duck Creek every $15 \mathrm{~s}$ for $240 \mathrm{~s}$. Bed shear stress was $6 \mathrm{~Pa}$ (twice the threshold for movement of the $4 \mathrm{~mm}$ median diameter gravel), and transport was almost entirely as bed load. Particles moved mainly by rolling and saltation, and rarely by sliding. The TESTFADE variance plot (Figures $2 \mathrm{a}$ and $2 \mathrm{c}$ ) shows sub-dispersive spreading at the intermediate time scale, consistent with experimental observations, and predicts super-dispersion at early time (Figure 2a). The detected particle mass (Figure 2f) decreases with time because: (1) approximately $10 \%$ of particles never moved from the starting point; (2) some particles left the $180 \mathrm{~cm}$-long detection window during the experiment; and (3) buried particles were unobservable. Similar to Case 1, observed particle mass was used to estimate the time parameters $\gamma=0.60$, $\beta=0.1$ second $^{\gamma-1}$, and $\lambda_{t}=0.002$ second $^{-1}$. The measured variance of particle displacement (Figure 2c) was then used to estimate the remaining parameters: $\alpha=1.7$, $\lambda_{x}=0.001 \mathrm{~cm}^{-1}, V=1.0 \mathrm{~cm} / \mathrm{s}$, and $D=2.0 \mathrm{~cm}^{\alpha} / \mathrm{s}$.

\subsection{Case 3: Bed Load Transport in Balmoral Canal}

[13] Nikora et al. [2002] videotaped the complete trajectories of 159 gravel particles moving across a $20 \mathrm{~cm} \times$ $23 \mathrm{~cm}$ patch of canal bed within one second. The Shields number was $\tau^{*} \approx 0.02$. Particles moved intermittently by rolling, sliding, and saltation. Here the jump size distribution is most likely to be heavy-tailed, due to the observed positive skewness of particle displacements, the lack of trapping, 

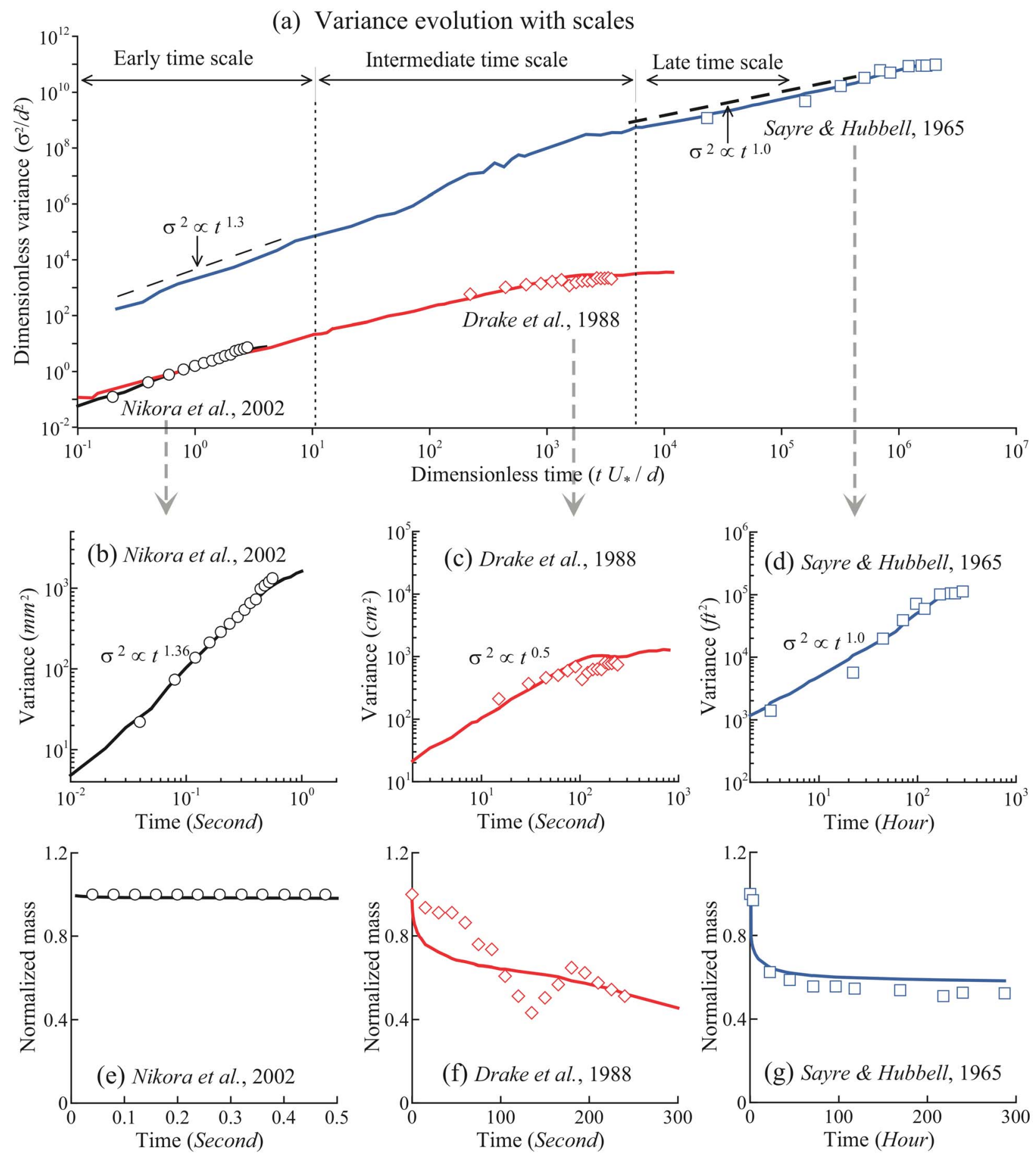

Figure 2. Moment evolution in bed load transport: Model results (solid lines) versus the measurements (symbols). (a) Variance of particle displacement in the dimensionless time of Nikora et al. [2002]. (b-d) The variance from Nikora et al. [2002], Drake et al. [1988], and Sayre and Hubbell [1965]. (e-g) The corresponding mobile mass for each case.

and long particle step lengths. During this early-time transport, the detected mass remains stable (Figure 2e), and the particle-displacement variance grows at a super-dispersive rate (Figure $2 \mathrm{~b}$ ). The best-fit parameters were: $\gamma=0.65, \beta=$ $0.02 \mathrm{~s}^{\gamma-1}$, and $\lambda_{t}=0.002 \mathrm{~s}^{-1}$, which preserves the mobile mass. The space index $\alpha=1.2$ was fitted by matching the slope of the observed variance (Figure $2 b$ ). The remaining parameters $\left(\lambda_{x}=1 \times 10^{-7} \mathrm{~mm}^{-1}, V=110 \mathrm{~mm} / \mathrm{s}\right.$, and
$D=130 \mathrm{~mm}^{\alpha} / \mathrm{s}$ ) were then fitted by matching the magnitudes of the observed variance.

\section{Discussion and Conclusion}

[14] Dispersion (and diffusion) phenomena can be grouped into three distinct classes: Fickian dispersion, where the observed plume variance grows linearly with time; 
anomalous super-dispersion, where the variance grows faster than linearly; and sub-dispersion, where variance grows slower than linearly. We applied the TESTFADE model to characterize sediment dispersion in three field experiments. Based on these results, we infer the underlying factors that control particle dispersion in each case. Inspired by Nikora et al. [2002], we consider three distinct time regimes of particle transport dynamics: an initial period of superdispersion resulting from the motion of exposed particles on the bed surface, a longer period of sub-dispersion resulting from episodic particle motion associated with turbulence, sheltering, or burial, and an eventual relaxation to Fickian dispersion.

[15] The microscale space-time random walk model ((1a) and (1b)) and the corresponding macroscale differential equation model ((3a) and (3b)) explicitly separate particle motion and resting phases. For small values of the tempering parameters $\lambda_{x}$ and $\lambda_{t}$, plume variance initially grows like $t^{2 / \alpha}$ and then eventually relaxes to linear Fickian growth. The early time scale is characterized by frequent particle motion with a wide distribution of step lengths. Plume variance growth is also slowed by the time process, essentially replacing $t$ by $t^{\gamma}$ in the intermediate time regime. If the motion process is still super-diffusive at intermediate time, then there is a competition between super-diffusive long jumps and sub-diffusive long rests, causing the plume variance to grow like $t^{2 \gamma / \alpha}$. Once the motion process becomes tempered, the ensemble behavior is sub-diffusive with a variance growth rate of $t^{\gamma}$. At late time, tempering of both the motion and resting processes produces asymptotic Fickian scaling where plume variance grows linearly with time. In practical terms, regular dispersion only occurs at spatial scales much longer than the particle jump lengths and time scales much longer than the intervals between transport events.

[16] Several mechanisms have been proposed for superdiffusive bed sediment motion at early times. Nikora et al. [2002] and Martin et al. [2012] attributed super-diffusion at early time to particle inertia, which leads to ballistic motion. Mobilized particles generally are subjected to increased hydrodynamic forces owing to reduced sheltering and exposure to higher stream velocities. This is particularly the case when particles undergo saltation and suspension. Hill et al. [2010] argued that power-law step lengths can occur when exponentially distributed step lengths for particles of different mobility are combined. However, finite limits of particle mobility can yield truncated or tempered power laws. Bradley et al. [2010] showed that superdiffusion can result from the inability to detect immobile buried particles. Their findings also indicate that mobile particles in sand-bed streams remain super-diffusive over long time scales. Many common experimental approaches facilitate super-diffusion, as introduction of tracer particles to the bed surface will make those particles much more mobile than average, resulting in super-diffusion until significant burial occurs. Conversely, if the most mobile particles are not sampled, e.g., because they pass out of the region of observation, then super-diffusive behavior will be underestimated.

[17] Several studies support the hypothesis that subdiffusive particle motion occurs because of sheltering or burial of particles in the bed [Nikora et al., 2002; Lajeunesse et al., 2010; Martin et al., 2012]. Brief immobilization is associated with sheltering on the bed surface and remobilization by turbulent flow fluctuations or particle impacts (e.g., from saltation) [Niño and García, 1996; Lajeunesse et al., 2010]. Intermediate burial times for near-surface material are related to bedform dynamics [Ferguson et al., 2002]. Long resting times are controlled by flow variations (e.g., frequency of floods) and large-scale morphodynamic processes, such as meandering [Aalto et al., 2008]. The highly detailed laboratory data set of Lajeunesse et al. [2010] showed that large jump sizes and long resting times can occur simultaneously. Martin et al. [2012] considered the resulting transition from initial super-diffusion at early time (governed by ballistic jumps) to sub-diffusion at a later time (governed by sheltering and burial). The same transitions from super-dispersion to sub-diffusion to Fickian diffusion have also been observed in movements of lipid molecules in phospholipid bilayers [Flenner et al., 2009]. The TESTFADE model presented here clarifies that the outcome of all mechanisms of infrequent, episodic particle motion is essentially similar: sub-diffusion owing to incomplete averaging over the periods of resting.

[18] No prior studies of sediment transport have described the full range of behavior from initial super-diffusion to intermediate sub-diffusion to late-time regular diffusion. Nikora et al. [2002] hypothesized three transport regimes, but believed that the late-time behavior would be subdiffusive. We show that the asymptotic behavior is regular diffusion, but our simulations clarify that regular diffusion only occurs when the observation time is much longer than the resting time. Such conditions can be obtained in laboratory experiments, where the system volume and thus the opportunity for burial is constrained, but it is not clear how commonly this behavior will occur in unconstrained natural systems. Beyond the opportunity for long-term burial in floodplains, deltas, or alluvial fans, non-stationarity in the underlying statistics of motion is expected because of downstream changes in streamflow and channel morphology. Under such conditions, the averaging required to achieve the asymptotic regular diffusion predicted by the model might occur only rarely. The model presented here can represent both late-time and non-stationary transport behavior, but field data are not currently available to support reasonable estimates of such long-term, large-scale sediment transport dynamics.

[19] The combined microscale-macroscale approach employed here provided a means to explicitly calculate the transition from initial super-diffusion to intermediate subdiffusion to late-time regular diffusion in bed sediment transport. This unifies prior observations of particle dispersion behavior in rivers, as it can now be seen that the disparate ensemble particle dynamics observed in different experiments reflect the extent of averaging associated with each set of observations. The model presented here explicitly predicts ensemble dynamics for any averaging scale, and thereby can be used to relate observations of sediment transport across scales, as illustrated in Figure 2. This approach should be useful for a wide range of geophysical and ecological phenomena that exhibit similar interplay of longdistance transport and episodic motion, such as ecological dispersal, pathogen transmission in rivers, nutrient export from watersheds, and large-scale geomorphodynamics associated 
with infrequent phenomena such as avalanches and turbidity currents.

[20] Acknowledgments. This work was partially supported by NSF grants DMS-1025417, DMS-1025486, DMS-0803360, EAR-0810270, and NIH grant R01-EB012079. We thank D. J. Jerolmack for helpful comments that significantly improved this paper.

[21] The Editor thanks the anonymous reviewers.

\section{References}

Aalto, R., J. W. Lauer, and W. E. Dietrich (2008), Spatial and temporal dynamics of sediment accumulation and exchange along Strickland River floodplains over decadal-to-centennial timescales, J. Geophys. Res., 113, F01S04, doi:10.1029/2006JF000627.

Baeumer, B., and M. M. Meerschaert (2010), Tempered stable Levy motion and transient super-diffusion, J. Comput. Appl. Math., 233, 2438-2448, doi:10.1016/j.cam.2009.10.027.

Bradley, D. N., G. E. Tucker, and D. A. Benson (2010), Fractional dispersion in a sand bed river, J. Geophys. Res., 115, F00A09, doi:10.1029/ 2009JF001268.

Chakrabarty, A., and M. M. Meerschaert (2011), Tempered stable laws as random walk limits, Statist. Probab. Lett., 81(8), 989-997.

Drake, T. G., R. L. Shreve, W. E. Dietrich, P. J. Whiting, and L. B. Leopold (1988), Bedload transport of fine gravel observed by motion-picture photography, J. Fluid Mech., 192, 193-217.

Einstein, H. A. (1937), Bed load transport as a probability problem, $\mathrm{PhD}$ thesis, Res. Inst. of Hydraul. Eng., Eidgenossische Tech. Hochschule, Zurich, Switzerland.

Ferguson, R. I., D. J. Bloomer, T. B. Hoey, and A. Werritty (2002), Mobility of river tracer pebbles over different timescales, Water Resour. Res., 38(5), 1045, doi:10.1029/2001WR000254.

Flenner, E., J. Das, M. C. Rheinstädter, and I. Kosztin (2009), Subdiffusion and lateral diffusion coefficient of lipid atoms and molecules in phospholipid bilayers, Phys. Rev. E, 79, 011907, doi:10.1103/PhysRevE.79.011907.

Hill, K. M., L. Dellangelo, and M. M. Meerschaert (2010), Heavy tailed travel distance in gravel bed transport: An exploratory enquiry, J. Geophys. Res., 115, F00A14, doi:10.1029/2009JF001276.
Jerolmack, D. J. (2011), Causes and effects of noise in landscape dynamics, Eos Trans. AGU, 92(44), 385-386.

Jerolmack, D. J., and D. Mohrig (2005), A unified model for subaqueous bed form dynamics, Water Resour. Res., 41, W12421, doi:10.1029/ 2005WR004329.

Lajeunesse, E., L. Malverti, and F. Charru (2010), Bed load transport in turbulent flow at the grain scale: Experiments and modeling, J. Geophys. Res., 115, F04001, doi:10.1029/2009JF001628.

Martin, R. L., D. J. Jerolmack, and R. Schumer (2012), The physical basis for anomalous diffusion in bed load transport, J. Geophys. Res., 117, F01018, doi:10.1029/2011JF002075.

Meerschaert, M. M., Y. Zhang, and B. Baeumer (2008), Tempered anomalous diffusion in heterogeneous systems, Geophys. Res. Lett., 35, L17403, doi:10.1029/2008GL034899.

Meerschaert, M. M., and A. Sikorskii (2012), Stochastic Models for Fractional Calculus, Stud. Math., vol. 43, de Gruyter, Berlin.

Niño, Y., and M. García (1996), Experiments on particle-turbulence interactions in the near wall region of an open channel flow: Implications for sediment transport, J. Fluid Mech., 326, 285-319.

Nikora, V. I., A. N. Sukhodolov, and P. M. Rowinski (1997), Statistical sand wave dynamics in one-directional water flows, J. Fluid Mech., $351,17-39$

Nikora, V., H. Habersack, T. Huber, and I. McEwan (2002), On bed particle diffusion in gravel bed flows under weak bed load transport, Water Resour. Res., 38(6), 1081, doi:10.1029/2001WR000513.

Rosiński, J. (2007), Tempering stable processes, Stoch. Proc. Appl., 117, $677-707$.

Samorodnitsky, G., and M. S. Taqqu (1994), Stable non-Gaussian random processes: Stochastic Models with infinite variance, 632 pp., Chapman and Hall, New York.

Sayre, W., and D. Hubbell (1965), Transport and dispersion of labeled bed material, North Loup River, Nebraska, U.S. Geol. Surv. Prof. Pap., 433-C, $48 \mathrm{pp}$.

Zhang, Y., and M. M. Meerschaert (2011), Gaussian setting time for solute transport in fluvial systems, Water Resour. Res., 47, W08601, doi:10.1029/2010WR010102. 